\title{
Searches for new particles at Colliders
}

\section{Fabiola Gianotti*}

CERN, EP Division, 1211 Genève 23, Switzerland

E-mail: 'fabiola.gianotti@cern.ch'

ABSTRACT: Most recent results from the searches for new particles at LEP, Tevatron and HERA, presented at the 2001 Summer Conferences, are reviewed. Prospects at future machines (LHC and Linear Colliders) are also discussed. The emphasis is put on the quest for Higgs bosons, Supersymmetry, Extra-dimensions and Flavour-Changing Neutral Current processes. In each case, the phenomenological framework, the experimental strategies, and the analysis methods are described, and the sensitivity and reach of the various machines are compared.

\section{Introduction}

The search for new particles is one of the most exciting, rapidly-evolving and prolific fields in today's high-energy experimental physics, motivated also by a rich spectrum of theoretical scenarios and predictions. Over the last years, the three operational high-energy Colliders, LEP, Tevatron and HERA, have explored the few hundred GeV energy range and set stringent bounds on various models. In addition, recently LEP has reported an exciting $2 \sigma$ hint in the search for a Standard Model (SM) Higgs boson of mass about $115 \mathrm{GeV}$, and the H1 experiment at HERA has observed an excess of $W$ 's produced in association with a high transverse momentum hadronic system.

Since 1996, i.e. since the beginning of its phase two, LEP has delivered an integrated luminosity of about $700 \mathrm{pb}^{-1}$ per experiment at a centre-of-mass energy above the $W$-pair production threshold. The machine performance in terms of both energy and luminosity went beyond any optimistic expectation. Most relevant to searches are the data recorded in the year 2000 (the last year of operation) at centre-of-mass energies above $206 \mathrm{GeV}$, about $130 \mathrm{pb}^{-1}$ per experiment, of which about $8 \mathrm{pb}^{-1}$ per experiment at the highest useful energy of $\sqrt{s} \simeq 208 \mathrm{GeV}$.

The Tevatron experiments CDF and D0 have collected about $110 \mathrm{pb}^{-1}$ each at $\sqrt{s}=$ $1.8 \mathrm{TeV}$ during Run 1. Run 2 has started in Spring 2001 at a centre-of-mass energy of

\footnotetext{
${ }^{*}$ Speaker.
} 
$2 \mathrm{TeV}$ and with a luminosity goal of up to $5 \cdot 10^{32} \mathrm{~cm}^{-2} \mathrm{~s}^{-1}$. Only results from Run 1 have been presented at this Conference.

At HERA, the $\mathrm{H} 1$ and ZEUS experiments have both collected about $135 \mathrm{pb}^{-1}$ until September 2000, at a centre-of-mass energy of up to $318 \mathrm{GeV}$. Data taking has started again in Autumn 2001 with an upgraded machine and a luminosity goal of $7 \cdot 10^{31} \mathrm{~cm}^{-2} \mathrm{~s}^{-1}$.

Most recent results from the searches for new particles at LEP, Tevatron and HERA, presented at the 2001 Summer Conferences, are reviewed in this paper ${ }^{1}$. Prospects at the CERN Large Hadron Collider (LHC) and at future Linear Colliders are also discussed briefly. For each topic, the phenomenological framework, the experimental strategies and the analysis methods are described, and the sensitivity and reach of the various machines are compared.

This paper is organised as follows. The quest for the SM Higgs boson, in particular the recent LEP hint, is described in section $\overline{2}_{i}$, problems related to the SM Higgs mechanism and possible solutions beyond the Standard Model are briefly recalled in section isi; the searches for Supersymmetry (SUSY), Extra-dimensions and Flavour-Changing Neutral Current (FCNC) processes are presented in sections 崔 to

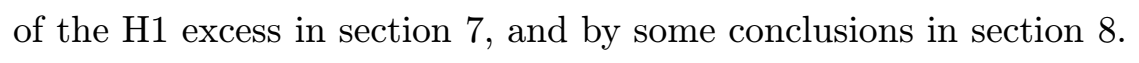

\section{Search for the Standard Model Higgs boson}

As of today, the only relevant results from the search for a SM Higgs boson come from LEP. Using the data collected until 1999, in which no signal had been found, the LEP experiments were able to set a lower limit on the Higgs mass of $107.9 \mathrm{GeV}$ at $95 \%$ C.L.. In the year 2000, when the machine was running at a centre-of-mass energy above $205 \mathrm{GeV}$, an excess of events was observed, consistent with the production of a Higgs boson of mass $\sim 115 \mathrm{GeV}$. At the end of data taking, on November 3rd 2000, the four experiments reported a combined excess of $2.9 \sigma$ [i] the 2001 Summer Conferences, the Higgs phenomenology at LEP and the search methods are briefly recalled.

At LEP, a SM Higgs boson is mainly produced in association with a $Z$ boson through the Higgsstrahlung process $e^{+} e^{-} \rightarrow Z^{*} \rightarrow Z H$. If the Higgs mass is indeed $\sim 115 \mathrm{GeV}$, about 35 events have been produced at LEP in the year 2000 at $\sqrt{s} \geq 206.6 \mathrm{GeV}$. In this mass range, the dominant Higgs decay modes are $H \rightarrow b \bar{b}$ (branching ratio $\sim 75 \%$ ) and $H \rightarrow \tau \tau$ (branching ratio $\sim 7 \%$ ). Therefore Higgs production at LEP should give rise to four main final states, addressed by as many dedicated searches: four jets final states (indicated here as $H q q$ ), if $H \rightarrow b \bar{b}$ and $Z \rightarrow q \bar{q}$, which is the channel with the largest sensitivity; two jets and missing energy final states $(H \nu \nu)$, if $H \rightarrow b \bar{b}$ and $Z \rightarrow \nu \bar{\nu}$; two jets and two leptons final states $(H \ell \ell)$, if $H \rightarrow b \bar{b}$ and $Z \rightarrow e e, \mu \mu$; and two jets and two taus final states $(q q \tau \tau)$, if either $H \rightarrow b \bar{b}$ and $Z \rightarrow \tau \tau$ or $H \rightarrow \tau \tau$ and $Z \rightarrow q \bar{q}$. It should be noticed that LEP is sensitive to most topologies arising from Higgs production and decay, unlike Hadron Colliders where fully hadronic final states are difficult to extract from the

\footnotetext{
${ }^{1}$ It would be impossible to describe in one paper the impressive amount of search studies and results reported at this Conference. The reader is therefore referred to other contributions to these Proceedings.
} 
background. At LEP, backgrounds arise mainly from $Z Z$ and $W W$ production, which yield events containing four jets, jets and leptons, jets and missing energy. In addition, $q \bar{q}$ production is dangerous in two cases: when final-state gluon radiation gives rise to a four-jet topology, and when initial-state photon radiation with the photon(s) lost in the beam pipe mimics the two jet plus missing energy topology. The cross-sections for these processes, measured by the L3 experiment as a function of energy, are displayed in figure together with the cross-section expected for a Higgs boson of mass $115 \mathrm{GeV}$. It can be seen that the main backgrounds are globally well understood, and that the needed rejection is at most a factor of $10^{3}$, unlike at Hadron Colliders where rejections of up to $10^{7}$ must be achieved. Such a rejection is obtained at LEP by using several handles, such as the presence of $b$-jets and of a $Z$-boson in the final state and the event kinematics.

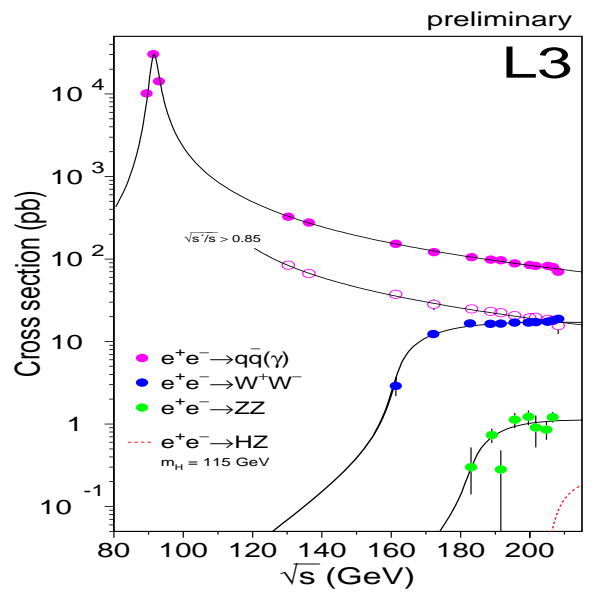

Figure 1: Cross-sections of several SM processes, as a function of the centre-of-mass energy, as measured by L3 (dots). The full lines show the theoretical prediction. The dotted line indicates the expected cross-section for a Higgs boson of mass $115 \mathrm{GeV}$.

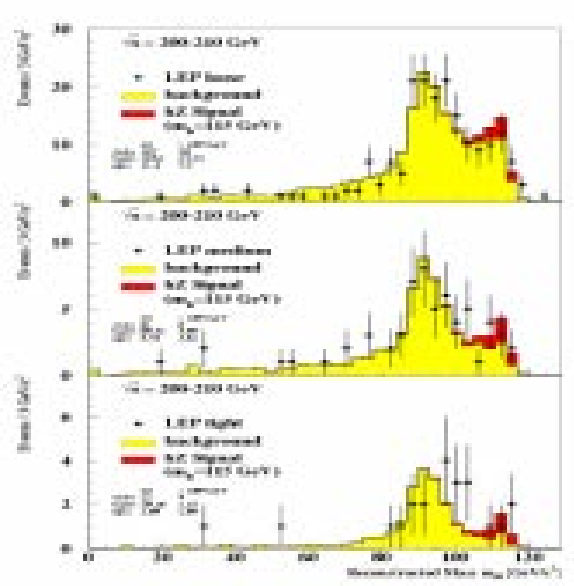

Figure 2: Reconstructed Higgs boson mass, as obtained from the year 2000 LEP data (dots with error bars) by applying a loose (top), a medium (middle) and a tight (bottom) selection [2ind. The expected SM background (light-shaded histogram) and Higgs signal for a mass of $115 \mathrm{GeV}$ (dark-shaded histogram) are also shown.

Figure $\bar{n}$ shows the reconstructed Higgs mass spectra from the data collected by the four LEP experiments in the year 2000 [2] 2$]$, after applying three special non-biasing selections: a loose selection, a medium selection, and a tight selection. When cuts are tightened, a more signal-like sample is selected, and a slight excess becomes visible at high masses. The magnitude of this excess is compatible with the low rate expected from a possible signal, also shown in the figure.

Although the reconstructed mass spectra are graphically intuitive and simple to understand, they are only one of several discriminating variables and by far do not contain all the information which can be used to distinguish a Higgs signal from the background processes. Other discriminating features are for instance the $b$-quark content of an event and its topology. In particular, for a signal at the limit of the kinematic reach of a machine, 
as is the case for a Higgs of mass $\sim 115 \mathrm{GeV}$ at LEP, the number of events expected is small and clear mass peaks can not be observed. It is therefore important to use at the best all the information contained in the data, in order to maximise the sensitivity to a potential signal.

For this reason the LEP experiments use a statistical estimator, the likelihood ratio $Q$, which allows all the relevant features of the observed candidates in the data to be compared with two hypotheses: that these candidates come from background only, and that they come from a mixture of signal and background. For a given hypothetical Higgs mass $m_{H}$, the likelihood ratio $Q$ is simply the ratio of the Poisson probabilities for the background-only hypothesis $L(b)$ and for the signal+background hypothesis $L(s+b)$ :

$$
Q\left(m_{H}\right)=\frac{L(s+b)}{L(b)}=\frac{e^{-(s+b)}}{e^{-b}} \prod_{i=1}^{N_{c a n d}} \frac{s_{i}+b_{i}}{b_{i}}
$$

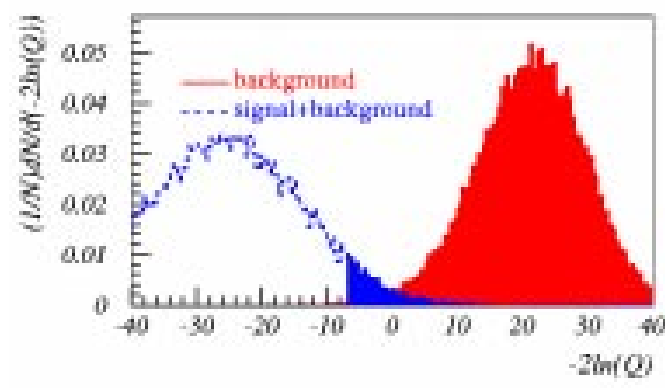

Figure 3: Examples of probability distributions for the statistical estimator $-2 \ln Q$, as expected from simulated background-only experiments (light-shaded) and from simulated signal+background experiments (histogram). The dark-shaded area defines $C L_{s+b}$, see text.

Here the product runs over the number of candidates $\left(N_{\text {cand }}\right)$ observed in the data, and $s_{i}$ and $b_{i}$ are the numbers of signal and background events expected with all the features (b-tagging, kinematics, reconstructed Higgs mass, etc.) of candidate $i$ in the data.

Numerically, it is more convenient to take the logarithm of $Q$ :

$$
-2 \ln Q\left(m_{H}\right)=2 s-2 \sum_{i=1}^{N_{\text {cand }}} \ln \left(1+\frac{s_{i}}{b_{i}}\right)
$$

where the ratio in the parenthesis is called the "weight" of candidate $i$.

Figure $\overline{3}$ is a schematic illustration of the expected distributions of $-2 \ln Q$ for two classes of simulated experiments: experiments observing only background, and experiments observing a signal on top of the background. If there is no signal in the data, $Q$ is small, and therefore the distribution of $-2 \ln Q$ for background-only experiments peaks at positive values. If there is a signal in the data, $Q$ is large, and the distribution of $-2 \ln Q$ for signal+background experiments is expected to be in the negative region. Therefore, if the value of $-2 \ln Q$ observed by an experiment is positive, then the background-only hypothesis is favoured, otherwise the signal+background hypothesis is favoured. To better quantify the compatibility of the observation with these two hypotheses, two numbers are defined. The background-only confidence level $\left(1-C L_{b}\right)$ is the integral of the $-2 \ln Q$ distribution for an ensemble of simulated background-only experiments from $-\infty$ to the observed value in the data, and gives the probability that such experiments are more signal-like than the observation. A small $1-C L_{b}$ indicates a signal-like observation. The signal+background confidence level $\left(C L_{s+b}\right)$ is the integral of the $-2 \ln Q$ distribution for an ensemble of simulated signal+background experiments from the observed value in the 
data to $+\infty$, and gives the probability that such experiments are more background-like than the observation. A large $C L_{s+b}$ indicates a signal-like observation.

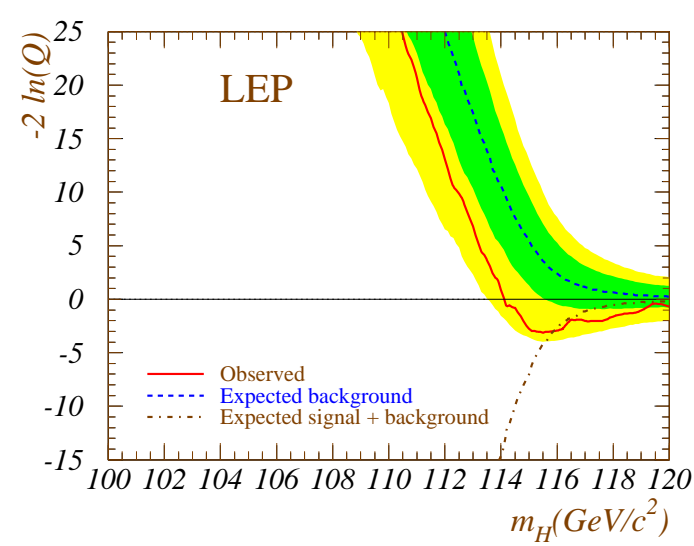

Figure 4: Distribution of the log-likelihood ratio $-2 \ln Q$ as a function of the Higgs test mass for the LEP data. The solid line shows the observation, the dashed line the median background expectation, and the dashdotted line the median signal+background expectation. The dark (light) shaded band indicates the $\pm 1 \sigma( \pm 2 \sigma)$ spread of the background expectation [2in].

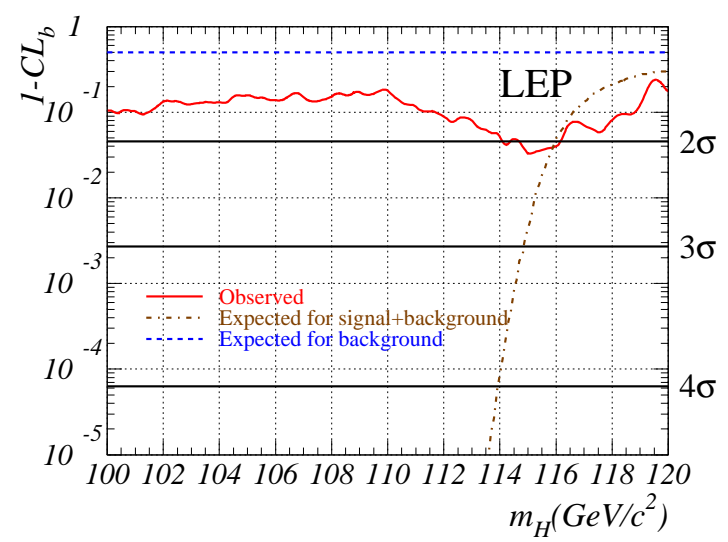

Figure 5: The background-only confidence level $1-C L_{b}$ of the LEP data as a function of the Higgs test mass. The solid line shows the observation, the dashed line the backgroundonly expectation, and the dash-dotted line the signal+background expectation $[\overrightarrow{2} i]$.

The results obtained at LEP are summarised in figure 霄. The observed distribution of $-2 \ln Q$ as a function of the Higgs test mass shows a minimum in the signal+background region, thereby indicating a deviation from the background-only hypothesis. The exact minimum is found for a mass of $115.6 \mathrm{GeV}$ and coincides with the expectation from a Higgs signal of the same mass. The excess is also visible from the small values of $1-C L_{b}$ (figure $\overline{p_{1}^{\prime}}$ ). In particular, for a mass of $115.6 \mathrm{GeV}$ the confidence level of the background-only hypothesis, i.e. the probability of a background fluctuation, is $3.5 \%$, which corresponds to an excess of $2.1 \sigma$. For this same mass value, the confidence level of the signal+background hypothesis $\left(C L_{s+b}\right)$ is $43 \%$. Both figures show that the excess is not a sharp minimum, but extends at lower masses. This behaviour is expected in the signal hypothesis and comes from mass resolution effects [2i].

There are several reasons why the significance of the excess has decreased from the $2.9 \sigma$ reported on November 3rd 2000, which was based on preliminary analyses, to the $2.1 \sigma$ presented at the 2001 Summer Conferences (table $\left.\underline{1}_{1}^{1}\right)$. The four experiments have included about $15 \mathrm{pb}^{-1}$ of data, which were not yet analysed on November 3rd, and only one new significant candidate has been found (by OPAL). ALEPH has improved the treatment of the correlation between the discriminating variables used for the calculation of $-2 \ln Q$. L3 has optimised the search analyses for the four-jet and missing-energy final states and has produced more Monte Carlo events at the correct centre-of-mass energies. As a consequence, the weight of the best L3 candidate, a missing-energy event, has decreased from 


\begin{tabular}{|c|c|c|}
\hline & November 3rd 2000 & 2001 Summer Conferences \\
\hline ALEPH & $3.4 \sigma$ & $3.0 \sigma[\overline{3} \overline{3}]$ \\
\hline DELPHI & $1-C L_{b}=0.68$ & $1-C L_{b}=0.77\left[\begin{array}{l}{\left[\begin{array}{l}1 \\
1\end{array}\right]} \\
1\end{array}\right]$ \\
\hline L3 & $1.8 \sigma$ & 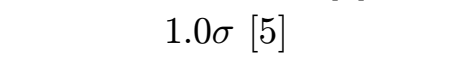 \\
\hline OPAL & $1.3 \sigma$ & $1.3 \sigma[\underline{\underline{6}}]$ \\
\hline LEP combined & $2.9 \sigma$ & $2.1 \sigma[\underline{2}]$ \\
\hline
\end{tabular}

Table 1: Significance of the events observed by the individual LEP experiments, and their combination, for a Higgs mass of $115 \mathrm{GeV}$, as reported on November 3rd 2000 and at the 2001 Summer Conferences.

$s / b=1.6$ to $s / b=0.7$ between November 2000 and Summer 2001. In addition, the data of all experiments have been reprocessed with refined calibrations, many systematic uncertainties (related, e.g., to the detector performance and to the knowledge of the backgrounds) have been scrutinised and studied in detail, and a lot of work has been done in the LEP Higgs working group [i2 $]$, in particular many cross-checks among the four experiments. It should be noticed that only the L3 result in table $\prod_{1}^{1}$ is final.

The sharing of the excess among the experiments can be deduced from tables

ALEPH is signal-like, with an excess of about $3 \sigma$ obtained with both a neural network based analysis and a simpler cut-based analysis [i]. hand, is background-like, whereas L3 and OPAL prefer slightly the signal+background hypothesis to the background-only hypothesis. This spread of results among the four experiments is not incompatible with the presence of a signal with these small statistics. Indeed, as shown in figure ${ }_{5}^{1-}$ the four experiments together, and not each of them individually, have a sensitivity at the level of $2 \sigma$. It is therefore not surprising that one of them is signal-like and another one background-like. Table ${ }_{-1}^{2}$ also shows that if the most signal-like experiment (ALEPH) is ignored, the combination of the other three experiments (DLO) is well compatible with the background-only hypothesis. However, the lower limit on the Higgs mass obtained by DLO, $m_{H}>114.8 \mathrm{GeV}$

\begin{tabular}{|c|c|c|}
\hline & $1-C L_{b}$ & $C L_{s+b}$ \\
\hline ALEPH & 0.002 & 0.94 \\
DELPHI & 0.87 & 0.02 \\
L3 & 0.24 & 0.47 \\
OPAL & 0.22 & 0.47 \\
\hline LEP & 0.035 & 0.43 \\
\hline DLO & 0.49 & 0.07 \\
ALO & 0.0037 & 0.83 \\
\hline
\end{tabular}

Table 2: For a Higgs test mass of $115.6 \mathrm{GeV}$, confidence levels of the background-only and of the signal+background hypotheses for the individual experiments and their combination. The results obtained by combinining DELPHI, L3 and OPAL (DLO) and ALEPH, L3 and OPAL (ALO) are also shown. at the $95 \%$ C.L., is not able to exclude the ALEPH excess at a mass of $\sim 115.6 \mathrm{GeV}$. If the most background-like experiment (DELPHI) is ignored, the combination of the other three experiments (ALO) has a probability to come from a background fluctuation of $0.4 \%$.

Some features of the fifteen most significant candidates for a Higgs test mass of $115 \mathrm{GeV}$ are listed in table 1 . ALEPH has three very pure four-jet events with a large $s / b$ ratio. The first two contribute about half of the observed LEP excess, whereas the rest of the excess is due to smaller weight events distributed among the four experiments and the decay 
channels. The large number of significant candidates in the $H q q$ channel is consistent with the signal hypothesis, since the four-jet final state provides the largest experimental sensitivity.

\begin{tabular}{|c|c|c|c|c|}
\hline Candidate & Experiment & Final state & $m_{H}^{r e c}(\mathrm{GeV})$ & $s / b$ at $115 \mathrm{GeV}$ \\
\hline 1 & ALEPH & $H q q$ & 114.3 & 4.7 \\
2 & ALEPH & $H q q$ & 112.9 & 2.3 \\
3 & ALEPH & $H q q$ & 110.0 & 0.9 \\
4 & L3 & $H \nu \nu$ & 115.0 & 0.7 \\
5 & OPAL & $H q q$ & 110.7 & 0.7 \\
6 & DELPHI & $H q q$ & 114.3 & 0.6 \\
7 & ALEPH & $H \ell \ell$ & 118.1 & 0.6 \\
8 & ALEPH & $q q \tau \tau$ & 115.4 & 0.5 \\
9 & ALEPH & $H q q$ & 114.5 & 0.5 \\
10 & OPAL & $H q q$ & 112.6 & 0.5 \\
11 & DELPHI & $H q q$ & 97.2 & 0.4 \\
12 & L3 & $H q q$ & 108.3 & 0.4 \\
13 & ALEPH & $H q q$ & 114.4 & 0.3 \\
14 & ALEPH & $H q q$ & 103.0 & 0.3 \\
15 & OPAL & $H \nu \nu$ & 104.0 & 0.3 \\
\hline
\end{tabular}

Table 3: Properties of the fifteen candidates contributing with the highest weight $s / b$ to $-2 \ln Q$ for a Higgs mass of $115 \mathrm{GeV}$. For each candidate, the experiment, the final state topology, the reconstructed Higgs mass and the weight are listed.

A graphical display of the event with the largest weight is shown in figure $1 \overline{6}_{1}^{1}$. It was collected by ALEPH at a centre-of-mass energy of $206.7 \mathrm{GeV}$, and contains four jets, two of which are well $b$-tagged (two well-reconstructed displaced vertices can be seen in figure (íi)'.

The reconstructed mass of the two $b$-jets, which come from the Higgs boson candidate, is $114.3 \pm 3.0 \mathrm{GeV}$, whereas the reconstructed mass of the two other jets before kinematic fit, $92.1 \mathrm{GeV}$, is very close to the nominal $Z$ mass. The event energy flow is very well reconstructed, as demonstrated also by the fact that the missing momentum vector points in the direction of one of the $b$-jets, where also a muon is observed, thereby indicating a semileptonic $b$ decay. A kinematic fit to the $H Z$ hypothesis gives a $\chi^{2}$ probability of the fit of about $53 \%$, whereas a fit to the $Z Z$ hypothesis gives a $\chi^{2}$ probability for the fit of $1 \%$ and a mass of $102 \mathrm{GeV}$ for one of the $Z$. The best background explanation would be $e^{+} e^{-} \rightarrow b \bar{b} \rightarrow b \bar{b} g g$ production, where the two gluons are radiated by the $b$-quarks in the final state. However, the two jets which are not $b$-tagged have energies $(\sim 45 \mathrm{GeV})$ and invariant mass $(92.1 \mathrm{GeV})$ compatible with coming from a $Z$ decay.

In conclusion, LEP has observed an excess of about $2 \sigma$ in the data collected in the year 2000 at a centre-of-mass energy above $205 \mathrm{GeV}$. If this excess is due to a signal from a SMlike Higgs boson, the preferred mass value is $115.6 \mathrm{GeV}$. The probability of a background fluctuation is $3.5 \%$. The lower limit on the Higgs mass obtained by combining the four experiments together is $m_{H}>114.1 \mathrm{GeV}$ at the $95 \%$ C.L., i.e., taking into account also 


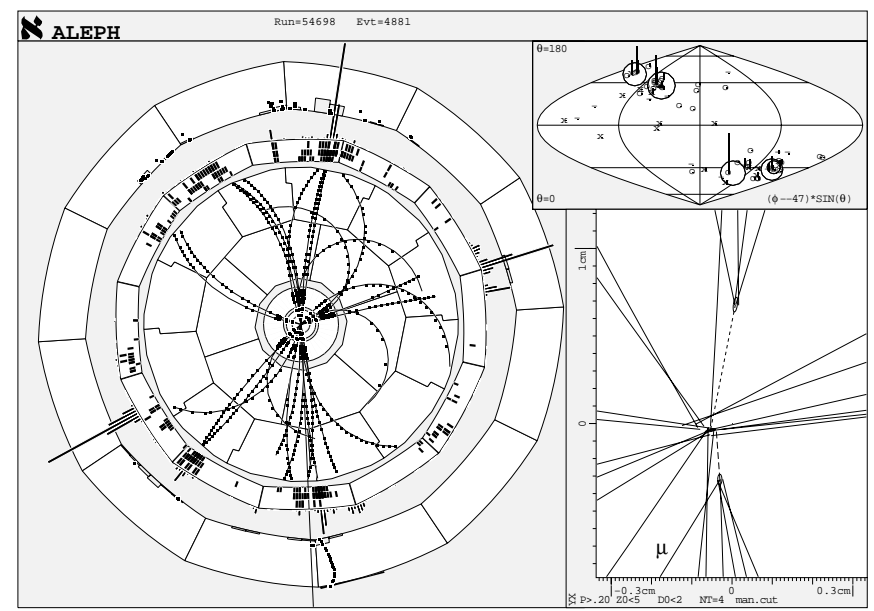

Figure 6: Display of the most significant LEP Higgs boson candidate, collected by ALEPH [i]] on June 14th 2000. The event is shown in the view transverse to the beam direction, in the $\theta-\phi \sin \theta$ view and in zoom of the vertex region.

previous LEP results, the mass range between zero and this limit is excluded today. It should be noted that in 1989, when LEP started to collect data, there was no compelling bound on the SM Higgs boson mass.

The Tevatron experiments CDF and D0 had little sensitivity to a SM Higgs signal in Run 1, but in Run 2 the luminosity is expected to be larger by a factor of up to 100, which

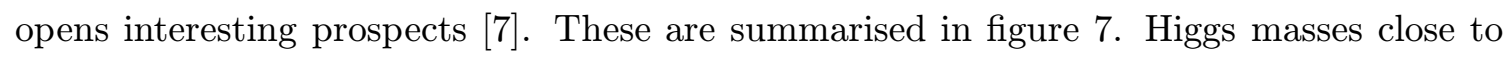

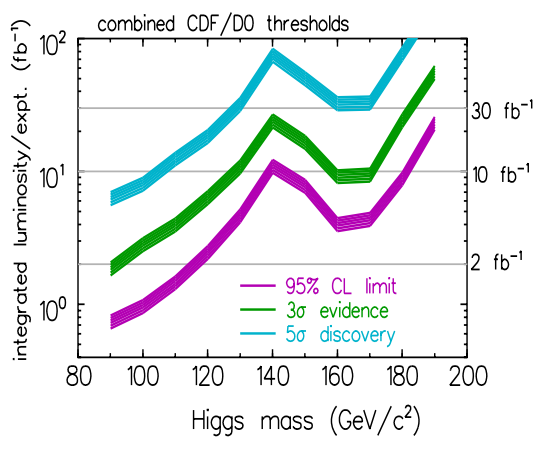

Figure 7: The integrated luminosities per experiment needed at the Tevatron Run 2 to exclude a SM Higgs boson at 95\% C.L. (lower band), to discover it at the $3 \sigma$ level (middle band) and at the $5 \sigma$ level (upper band), as a

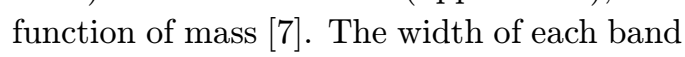
indicates the systematic uncertainty.

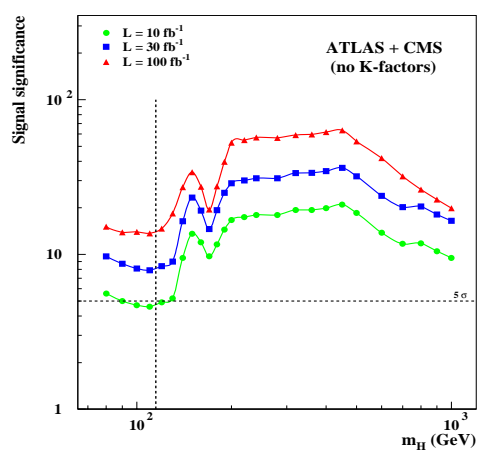

Figure 8: Expected signal significance as a function of the Higgs mass at the LHC for integrated luminosities of $10 \mathrm{fb}^{-1}$ (dots), $30 \mathrm{fb}^{-1}$ (squares) and $100 \mathrm{fb}^{-1}$ (triangles) per experiment. The vertical line indicates the LEP limit.

$115 \mathrm{GeV}$ can be excluded at the $95 \%$ C.L. with an integrated luminosity of $2 \mathrm{fb}^{-1}$ per 
experiment, which should be collected by 2003. After a luminosity upgrade, the machine could deliver, by the end of 2004 , the $5 \mathrm{fb}^{-1}$ needed for a $3 \sigma$ observation for $m_{H} \sim 115 \mathrm{GeV}$, and, by the end of 2007 , the $15 \mathrm{fb}^{-1}$ required for a $5 \sigma$ discovery up to masses of $\sim 120 \mathrm{GeV}$ or for a $95 \%$ C.L. exclusion up to masses of $\sim 185 \mathrm{GeV}$. Discovery for masses larger than $120 \mathrm{GeV}$ would require much more luminosity than $15 \mathrm{fb}^{-1}$ and looks therefore difficult. The most sensitive search channel at the Tevatron is $W H \rightarrow \ell \nu b \bar{b}$.

On the time scale of 2007 the LHC should become fully operational. The most recent schedule foresees a first physics run between August 2006 and February 2007, which should allow the ATLAS and CMS experiments to collect both about $10 \mathrm{fb}^{-1}$. As shown in figure with this integrated luminosity the two experiments could obtain a combined $5 \sigma$ significance for $m_{H}$ values close to the LEP limit. In this mass region, which is the most difficult one at the LHC, the sensitivity is provided by two complementary channels: $H \rightarrow \gamma \gamma$, which requires excellent electromagnetic calorimetry, and $t \bar{t} H$ production with $H \rightarrow b \bar{b}$, which requires mainly excellent b-tagging capabilities. For larger masses Higgs discovery is easier, unlike at the Tevatron, thanks to the very clean $H \rightarrow 4 \ell$ final state.

The year 2007 could therefore be a very exciting one since, if the Higgs mass is indeed close to $115 \mathrm{GeV}$, both machines, the Tevatron and the LHC, may have enough luminosity for a $5 \sigma$ discovery. Although it is obviously not easy to tell today who will discover the Higgs first, some comparison of the potential of these two Colliders can be made. At the LHC the expected signal rate is a factor of 10-100 (depending on the channel) larger than at the Tevatron, but the signal-to-background ratio is a factor of about five smaller. For the same Higgs mass (e.g. $115 \mathrm{GeV}$ ) and integrated luminosity (e.g. $10 \mathrm{fb}^{-1}$ ) the discovery potential is similar $(\sim 5 \sigma)$ at both machines. However, the LHC studies are quite conservative [i 8 i] in terms of assumed cross-sections, analysis methods, detector performance. Had the more optimistic approach of the Tevatron studies been adopted, the LHC discovery potential would increase from $5 \sigma$ to about $7 \sigma$ for the above-mentioned Higgs mass and luminosity. On the other hand, it will take a lot of time to understand complex detectors like ATLAS and CMS. Finally the main questions are, for the LHC if the machine and the experiments will indeed be ready by 2006, and for the Tevatron if the discovery luminosity of $15 \mathrm{fb}^{-1}$ will indeed be obtained by 2007 , given that a three times more intense $\bar{p}$ beam is needed to achieve this goal.

As already mentioned, for masses larger than $120 \mathrm{GeV}$ the LHC has no competition and should also be able to perform precise measurements of some of the Higgs properties "isin. However, a detailed investigation of the Higgs sector, including the measurements of the various branching ratios and couplings to the percent level, of the Higgs self-couplings and

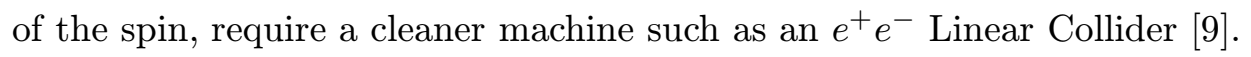

\section{Physics beyond the Standard Model}

It is well known that the SM Higgs mechanism entails some problems and therefore opens

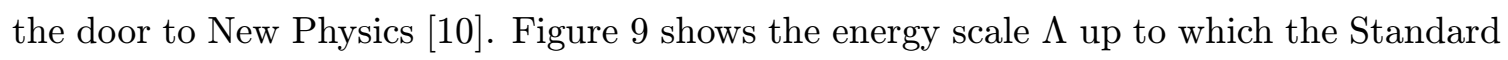
Model is valid, as a function of the Higgs mass. Although there are big uncertainties in these predictions [i] ${ }_{i}^{i} \overline{1}_{1}$, one can qualitatively conclude that, if the Higgs mass is close to 


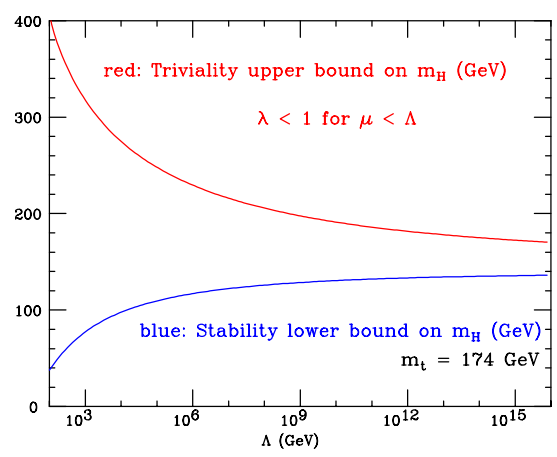

Figure 9: Theoretical bounds on the Higgs boson mass (in $\mathrm{GeV}$ ) as a function of energy $(\Lambda)$. The region above the top curve is forbidden because the Higgs self-coupling diverges, the region below the bottom curve is forbidden because the electroweak vacuum is unstable.

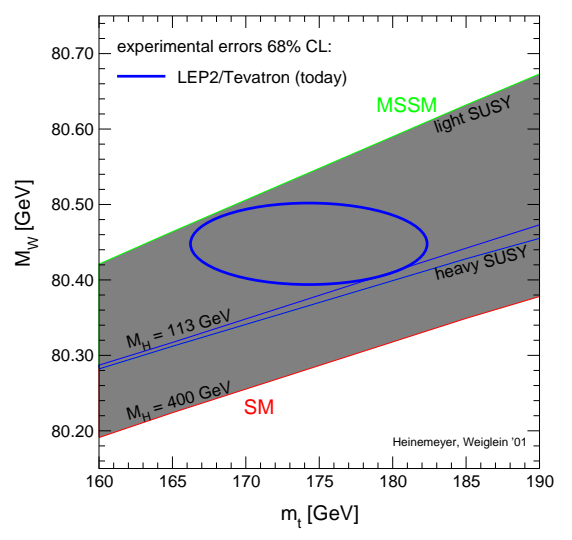

Figure 10: The $68 \%$ C.L. experimental contour in the plane $W$ mass versus top mass. The SUSY prediction is given by the top (lighter) band, whose size is determined by the allowed range of SUSY particle masses; the $\mathrm{SM}$ prediction for $m_{H} \leq 400 \mathrm{GeV}$ is given by the bottom (darker) band.

the LEP limit, then the Standard Model is expected to break down at an energy scale of about $10^{6} \mathrm{GeV}$, which means that New Physics should appear at this scale or below. On the other hand, if the Higgs mass is in the range 130-180 GeV, then the Standard Model technically works up to the Grand Unification scale. This scenario, which is not experimentally very attractive, introduces two additional problems: the huge difference between the electroweak scale and the Planck scale (17 orders of magnitude), the so-called "hierarchy problem"; and the fact that, due to radiative corrections, the mass of the Higgs boson, which is the only scalar of the theory, can become as heavy as $\Lambda$, and a lot of fine-tuning is needed to bring it down to the electroweak scale, the so-called "naturalness problem".

Three main solutions [i] $\left[\begin{array}{l}{[} \\ 0\end{array}\right]$ have been proposed to solve, at least in part, the hierarchy and the naturalness problems, which are related (but not exactly identical) issues:

- Supersymmetry predicts new particles at the TeV scale or below, which through radiative corrections stabilise the Higgs mass. Therefore the naturalness problem is solved, but the hierarchy problem is a priori not solved because there can still be a big gap between the SUSY scale and a more fundamental (higher) scale of the theory. No new interactions are introduced in addition to the SM gauge interactions.

- Technicolour predicts new strong interactions which break dynamically the electroweak symmetry. The naturalness problem is solved because the scalar Higgs is removed. The hierarchy problem is also solved because, like in QCD, the relation between the Technicolour scale and a more fundamental (higher) scale in the theory is exponential. New particles are predicted at the $\mathrm{TeV}$ scale.

- Extra-dimension theories predict the existence of additional spatial dimensions. In 
some models, these additional dimensions allow the gravity scale to be lowered down to the electroweak scale, thereby solving, at least in part, the hierarchy problem. New particles, e.g. excited gravitons, should appear at the $\mathrm{TeV}$ scale or below.

In all the above cases New Physics is predicted at the $\mathrm{TeV}$ scale, which strongly motivates machines like the LHC able to explore this energy range.

In this paper, only experimental results from searches for SUSY and Extra-dimensions are presented.

\section{Supersymmetry}

The numerous motivations and indications in favour of these theories [i] $\left.{ }_{1}^{[} \overline{0}\right]$ will not be

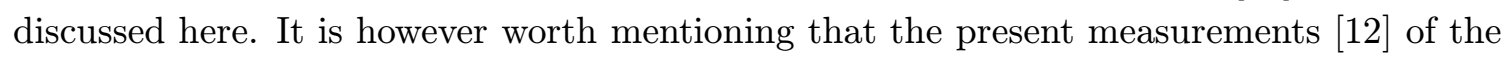
top mass (from Tevatron) and of the $W$ mass (mainly from LEP and Tevatron) are in excellent agreement with the SUSY prediction, as shown in figure i $1 \overline{0}^{\prime}$.

Many SUSY models exist, e.g. Supergravity, Gauge-Mediated SUSY-Breaking, AnomalyMediated SUSY-Breaking, which differ in the way SUSY breaking is transmitted to our world, and which predict different phenomenologies and experimental signatures. Here only results obtained in the framework of Supergravity models are discussed.

In the Minimal Supersymmetric extension of the Standard Model (MSSM) the physical spectrum consists of: squarks $(\tilde{q})$ and sleptons $(\tilde{\ell})$, which are the scalar partners of quarks and leptons; the gluino $(\tilde{g})$, which is the fermionic partner of the gluon; two charginos $\left(\chi_{1,2}^{ \pm}\right)$and four neutralinos $\left(\chi_{1,2,3,4}^{0}\right)$, which are mixtures of the fermionic partners of the electroweak and Higgs fields. There are two Higgs doublets in the theory, which give rise to five physical Higgs states: $h, H, A, H^{ \pm}$.

In order to reduce the number of parameters of the theory, a more constrained framework is often used (constrained MSSM, CMSSM), where all sfermions ( $\tilde{q}$ and $\tilde{\ell}$ ) have a common mass $m_{0}$ at the Grand Unification scale, and all gauginos $\left(\tilde{g}, \chi_{1,2}^{ \pm}, \chi_{1,2,3,4}^{0}\right)$ have a common mass $m_{1 / 2}$ at the Grand Unification scale. There are also a common trilinear mass paramater $\left(A_{0}\right)$ and a Higgsino mass parameter $(\mu)$. At the tree level the Higgs sector is described by two parameters, the mass of one of the Higgs bosons (e.g. $m_{A}$ ) and $\tan \beta$, which is the ratio of the vacuum expectation values of the two Higgs doublets. In more constrained models than the CMSSM, like mininal SUGRA, the parameters $m_{0}$ and $m_{A}$ are not independent and only the sign of $\mu$ is free.

There is a quantum number in the theory, R-parity, which takes the values of +1 for SM particles and -1 for SUSY particles (sparticles). If R-parity is conserved, as it is assumed here, SUSY particles are produced in pairs, and the Lightest Supersymmetric Particle (LSP), to which all SUSY particles eventually decay, is stable. The LSP is also required to be neutral and weakly-interacting for cosmological reasons, and therefore escapes experimental detection leading to the celebrated missing energy signature for Supersymmetry. In most models the LSP is the lightest neutralino $\left(\chi_{1}^{0}\right)$. 


\subsection{Searches for the MSSM Higgs bosons}

A distinctive feature of Supersymmetry is that the $h$ boson is light. Indeed, irrespective of the model, i.e. irrespective of the SUSY-breaking mechanism, the $h$ mass is predicted to be smaller than $\sim 135 \mathrm{GeV}$, except in very general models with additional fields [i] where (still) $m_{h} \leq 190 \mathrm{GeV}$. Hence, a light Higgs boson, as favoured by the electroweak data $[\overline{1} \overline{1} \overline{2}]$, is natural in Supersymmetry.

Searches at LEP have already excluded a good part of the allowed $h$ mass range [i] inin]. More precisely, since the $h$ mass increases (through radiative corrections) with increasing mixing between the SUSY partners of the left-handed and right-handed top quarks $\left(\tilde{t}_{L}\right.$, $\left.\tilde{t}_{R}\right)$, the scenario where there is no mixing between $\tilde{t}_{L}$ and $\tilde{t}_{R}$ has been almost fully ruled out by LEP since $m_{h}<115 \mathrm{GeV}$ in this case. On the other hand, the SUSY parameters can be chosen in such a way as to maximise the value of the $h$ mass, thereby leading to the more conservative " $m_{h}-$ max" scenario. For this case, the most recent LEP results are presented in figure $1 \overline{1} \overline{1}$, as a function of the parameters $m_{A}$ and $\tan \beta$.

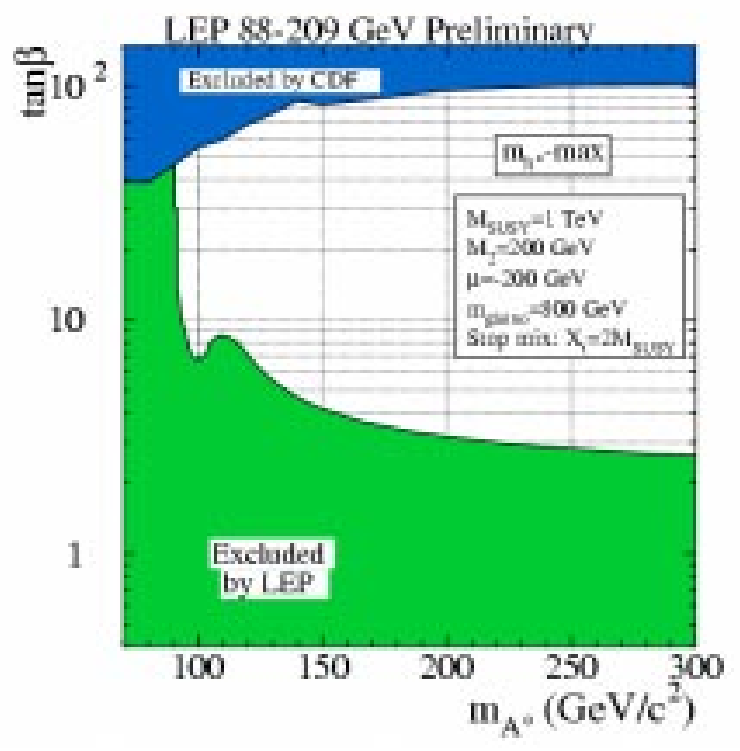

Figure 11: The region of the MSSM plane $m_{A}$-tan $\beta$ excluded by LEP at the $95 \%$ C.L. in the " $m_{h}-$ max" scenario [1 1 4]. The exclusion obtained by the CDF experiment in Run 1 is also shown [1는.

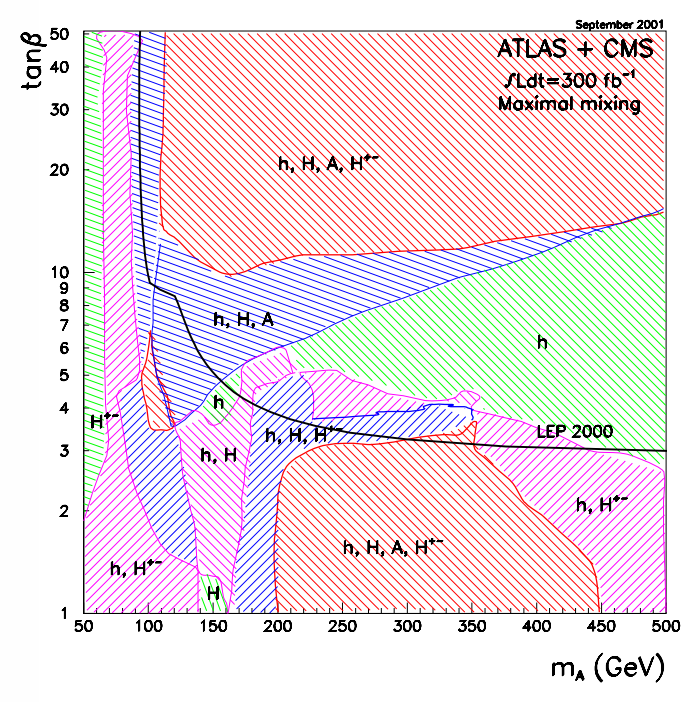

Figure 12: Regions of the MSSM plane $m_{A^{-}}$ $\tan \beta$ where the various SUSY Higgs bosons can be discovered at $\geq 5 \sigma$ at the LHC through their decays into SM particles, in the

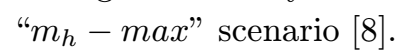

At LEP, only two SUSY Higgs channels are accesible: the associated production $h Z$ and the associated production $h A$. The other SUSY Higgs bosons are in most cases too heavy to be produced. The $h Z$ process is relevant mainly in the region $m_{A}>100 \mathrm{GeV}$, and gives rise to similar final states as $H Z$ production in the Standard Model. The $h A$ process is relevant in the region $m_{A}<100 \mathrm{GeV}$, and gives rise mainly to final states with four $b$-jets (when both Higgs bosons decay to $b \bar{b}$ pairs) or with two $b$-jets and two taus. As shown in figure ${ }_{1}^{1} \overline{1}_{1}^{\prime}$, searches for $h A$ production at LEP have excluded the region $m_{A}<91.9 \mathrm{GeV}$ at 
95\% C.L.. In the region at large $m_{A}$, the limit on the $h$ mass obtained from $h Z$ searches is, as in the $\mathrm{SM}$ case, $m_{h}>114.1 \mathrm{GeV}$. The latter can be translated into a lower limit on $\tan \beta$, as shown in the figure, since the $h$ mass increases with increasing $\tan \beta$. Namely, the region $0.5<\tan \beta<2.4$ is excluded at $95 \%$ C.L. in the " $m_{h}-$ max" scenario and for the central value of the measured top mass $(174.3 \mathrm{GeV})$. In the same scenario, this exclusion reduces to $0.6<\tan \beta<1.9$ in the more conservative case $m_{\text {top }}=179 \mathrm{GeV}$ (corresponding to $+1 \sigma$ from the central measured value). The experimental exclusion of the low $\tan \beta$ region of the MSSM parameter space is an important legacy from LEP.

As shown in figure $11 \overline{1} 1$, the CDF results from Run 1 [1] LEP exclusion. Indeed, Tevatron has enough centre-of-mass energy to produce any neutral MSSM Higgs boson in association with a $b \bar{b}$ pair $(h b \bar{b}, H b \bar{b}$ and $A b \bar{b})$ up to masses of about $300 \mathrm{GeV}$. These processes have rates which are strongly enhanced at large $\tan \beta$, and could therefore been observed with the data sample collected in Run 1. Since the Higgs bosons decay mainly into $b \bar{b}$ pairs, the expected final states contain four $b$-jets, to which only the CDF experiment was sensitive in Run 1 , because a very efficient $b$-tagging is required. The region between the CDF and the LEP contours is not excluded today because LEP has not enough centre-of-mass energy and CDF not enough luminosity. On the other hand, in Run 2 the Tevatron experiments should be able to explore the full MSSM Higgs plane at least at $95 \%$ C.L. [iiㅎ.

Discovery of at least one SUSY Higgs boson at the $\geq 5 \sigma$ level should be granted at the LHC, even with a small amount of luminosity $\left(10 \mathrm{fb}^{-1}\right.$ per experiment). Figure shows that over a good part of the parameter space several Higgs bosons are accessible at the LHC. The exception is the region at large $m_{A}$ and moderate $\tan \beta$, just above the LEP limit, where only $h$ can be discovered at the LHC unless the heavier Higgs bosons have observable decays into SUSY particles (e.g. charginos or neutralinos). The LHC may therefore miss the heavy part of the MSSM Higgs spectrum. Complete and modelindependent observation of this part of the spectrum may require a very high-energy Lepton Collider $(\sqrt{s} \geq 2 \mathrm{TeV})$.

\subsection{Searches for SUSY particles}

The expected SUSY phenomenology and the physics environment, and therefore the search strategies and the physics potential, are quite different at LEP (or, more generally, at Lepton Colliders) and at the Tevatron (or, more generally, at Hadron Colliders):

- At LEP, all kinematically accessible sparticles (except gluinos) are expected to be pair-produced more or less democratically through the $s$-channel with $\gamma / Z$ exchange. At the Tevatron, on the other hand, the production of $\tilde{q} \tilde{q}, \tilde{g} \tilde{g}, \tilde{q} \tilde{g}$, which is mediated by strong interactions, is expected to dominate by far over other (electroweak) processes.

- At the LEP energies, direct decays to the LSP are expected to dominate, e.g. $\tilde{\ell} \rightarrow$ $\ell \chi_{1}^{0}, \tilde{q} \rightarrow q \chi_{1}^{0}$. Therefore the main topology arising from the pair production of sparticles should be quite simple: two acoplanar objects (e.g. leptons, jets) plus 
missing energy produced by the escaping neutralinos. At Hadron Colliders, on the other hand, squarks and gluinos, which must be quite heavy given the present experimental limits (see below), are expected to decay through multi-step cascades, e.g. $\tilde{g} \rightarrow q \tilde{q} \rightarrow q q \chi_{2}^{0} \rightarrow q q Z \chi_{1}^{0}$. These cascade decays give rise to very crowded final states with many high- $p_{T}$ jets and leptons, plus large missing transverse energy.

- At LEP, Standard Model backgrounds (e.g. $W W$ and $Z Z$ production, $\gamma \gamma$ interactions) are not a big concern. As a consequence, the experiments are sensitive to almost all kinematically accessible sparticles, to almost all decay modes (including fully hadronic final states), and even to very modest $(\sim \mathrm{GeV})$ energy depositions in the detector, such as those expected if the mass difference $\Delta m$ between the produced sparticle and the LSP is small. The Tevatron experiments, on the other hand, are mainly sensitive to squarks and gluinos, which have a large cross-section and give rise to spectacular signatures used to reject the huge backgrounds (QCD multijet production, $W / Z+$ jets, etc.). Furthermore, the low $\Delta m$ region is not accessible, because a large amount of visible energy $(>>10 \mathrm{GeV})$ is needed to trigger the experiments and reject the backgrounds.

- As a consequence of the above points, at LEP the mass reach is limited mainly by the available centre-of-mass energy and luminosity rather than by the physics environment. In addition, by combining several searches it is possible to cover almost all corners of the kinematically accessible parameter space, and therefore to derive absolute mass limits (i.e. valid for any choice of the parameters) within constrained models. Tevatron, on the other hand, has a huge mass reach for squarks and gluinos (up to $\sim 300 \mathrm{GeV}$ in Run 1), but the kinematically accessible parameter space can not be fully covered (e.g. there is no sensitivity to the small $\Delta m$ values), and therefore absolute mass limits can not be established.

A few examples which illustrate the above points are discussed below. More details

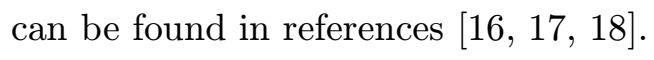

\subsubsection{Sleptons}

Despite the simple topology expected in the final state, i.e. two acoplanar leptons accompanied by missing energy, slepton pair production is not observable at the Tevatron because of the small signal-to-background ratio. Therefore the present experimental limits come mainly from LEP. Searches for acoplanar leptons in the data collected by the four experiments up to $\sqrt{s} \simeq 208 \mathrm{GeV}$ have found no significant deviations from the SM expectation (the dominant background is $W W \rightarrow \ell \nu \ell \nu$ production). A $20 \%$ excess of acoplanar $\tau \tau$ events observed in the '98 and '99 data [1] $\left.{ }^{1} 6\right]$ has not been confirmed by the year 2000 data. The derived mass limits, shown in figure 13 about $100 \mathrm{GeV}$ for selectrons (which benefit from a larger production cross-section). Some of these limits will remain valid until the LHC era. 


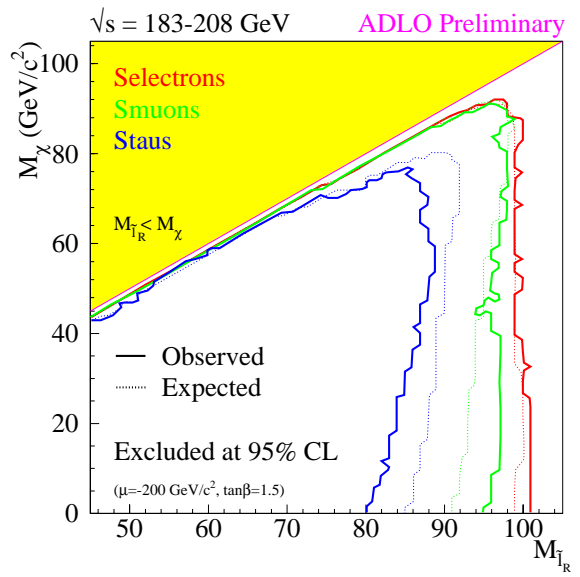

Figure 13: Regions of the plane LSP mass versus slepton mass excluded at 95\% C.L. by LEP [1] perimental limits on (from left to right) the stau mass, the smuon mass and the selectron mass. The dashed lines indicate the corresponding expected limits. The shaded region is theoretically forbidden.

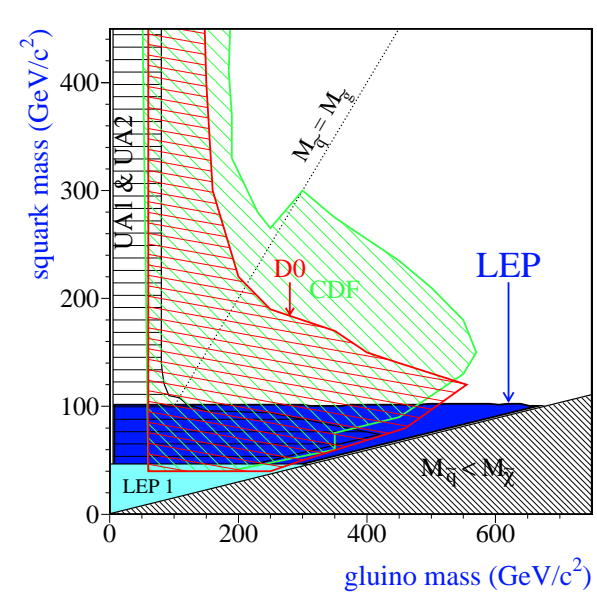

Figure 14: Regions of the plane squark mass versus gluino mass excluded at

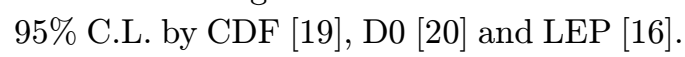
The hatched region at the bottom right corner is theoretically forbidden.

\subsubsection{Squarks and gluinos}

In contrast to the slepton case, Tevatron has the largest discovery potential for squarks and gluinos. The signature expected from the pair production of these sparticles consists of large missing transverse energy (from the escaping LSP) plus several jets and/or leptons coming from the more-or-less long cascade decays. One of the main experimental difficulties in these searches is to understand the missing transverse energy distribution in the data, which can receive large contributions from instrumental effects such as badly-measured jets in QCD multijet events.

The most stringent limits on the $\tilde{q}$ and $\tilde{g}$ masses, shown in figure ', $\overline{1} \overline{4}$, , come from a recent CDF search for events with multijet plus missing transverse energy [i $\left[\begin{array}{l}i \\ \overline{9}\end{array}\right]$. The highest mass reach, up to $\sim 300 \mathrm{GeV}$, is obtained if squarks and gluinos are mass-degenerate. The lower limit on the gluino mass valid for any value of the squark mass is $195 \mathrm{GeV}$. Figurei 1 is shows that the LEP experiments have a much more limited reach on the $\tilde{q}$ mass. However, unlike the Tevatron experiments, they are sensitive to mass differences between the squark and the LSP below $25 \mathrm{GeV}$ (such small $\Delta m$ values occur for large values of the gluino mass and small values of the squark mass). This is a nice example of complementarity between the two machines.

\subsubsection{Charginos}

LEP has the largest and most model-independent sensitivity to the production of these 
sparticles. The process $e^{+} e^{-} \rightarrow \chi^{+} \chi^{-}$, followed by $\chi^{ \pm} \rightarrow W^{*} \chi_{1}^{0}$ or $\chi^{ \pm} \rightarrow \ell \tilde{\nu} \rightarrow \ell \nu \chi_{1}^{0}$, is expected to give rise to final states containing jets plus missing energy, or jets plus one lepton plus missing energy, or acoplanar leptons. By looking for these topologies, the LEP experiments have been able to rule out charginos with masses smaller than $103.6 \mathrm{GeV}$, a bound which is only a few hundreds $\mathrm{MeV}$ below the kinematic limit for chargino pair production. This bound, which is valid over a large region of the parameter space, is deteriorated by a few $\mathrm{GeV}$ in two cases. If the common scalar mass $m_{0}$ is small, sneutrinos are light, and the chargino production cross-section is reduced by the negative interference between the $t$-channel with sneutrino exchange and the $s$-channel with $\gamma / Z$ exchange.

The second case is when the mass difference $\Delta m$ between the chargino and the LSP is small (figure $\left.1 \overline{1} \underline{1}_{1}^{\prime}\right)$. For $\Delta m>4-5 \mathrm{GeV}$ the standard searches mentioned above are used. For $\Delta m<100 \mathrm{MeV}$, charginos are stable, and therefore can be discovered or excluded by looking for anomalous ionisation (as expected from heavy stable charged particles) in the tracking detectors of the LEP experiments. For intermediate values of $\Delta m$, charginos decay into very soft hadrons, and a dedicated search has been developed which requires a hard initial-sate radiation (ISR) photon, to trigger the experiment and re-

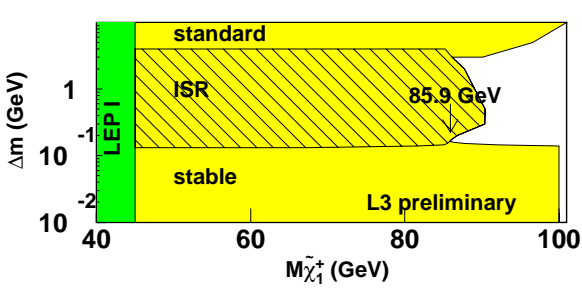

Figure 15: The 95\% C.L. lower limit on the chargino mass obtained by the L3 experiment [21]', as a function of the difference $\Delta m$ between the chargino and the LSP masses, in the most difficult low- $\Delta m$ region (see text).

ject the background, accompanied by a small amount of additional visible energy. Figure ${ }_{1}^{1} \overline{1} \bar{s}_{1}^{\prime}$ shows that the lower limit on the chargino mass obtained for any $\Delta m$ value $(85.9 \mathrm{GeV}$ in the case of the L3 experiment) is only $20 \mathrm{GeV}$ below the kinematic limit, thus demonstrating that LEP is able to address the most difficult topologies.

\subsubsection{Limit on the LSP mass}

A very important legacy from LEP is the absolute limit on the mass of the lightest neutralino, which has important cosmological implications because this sparticle is considered today the best candidate for the universe cold dark matter.

Since the direct production of $\chi_{1}^{0}$ pairs is not observable, an indirect limit is obtained from the interplay of the exclusion domains in the parameter space provided by other searches. This is possible because within constrained models, such as the CMSSM discussed here, the various sparticle masses are related. The result is given in figure $1 \overline{6}_{\mathfrak{v}}^{\prime \prime}$ which shows the lower limit on the neutralino mass as a function of the parameter $\tan \beta$. As previously mentioned, SUSY Higgs searches at LEP rule out the low $\tan \beta$ region. At larger $\tan \beta$ bounds from chargino and slepton searches also contribute. The absolute limit, $m\left(\chi_{1}^{0}\right)>45.6 \mathrm{GeV}$ at $95 \%$ C.L., is found asymptotically for $\tan \beta \geq 20$ and small $m_{0}$, in the region (named "corridor") where charginos decay into $\ell \tilde{\nu}$ pairs, and charginos and sneutrinos are almost mass-degenerate. In this case $\chi^{+} \chi^{-}$searches become ineffective because the two leptons in the final state are too soft to be efficiently detected. However, for small $m_{0}$ values sleptons should be accessible at LEP, and the negative results from slepton 
searches provide exclusion of part of these regions and hence the above-mentioned limit on the LSP mass. In the more constrained minimal SUGRA model, this limit improves to about $60 \mathrm{GeV}$ for $m_{\mathrm{top}}=175 \mathrm{GeV}$. More details on the method used to derive the LSP limit, as well as a discussion of the impact of mixing in the stau sector, can be found in [2]2in]

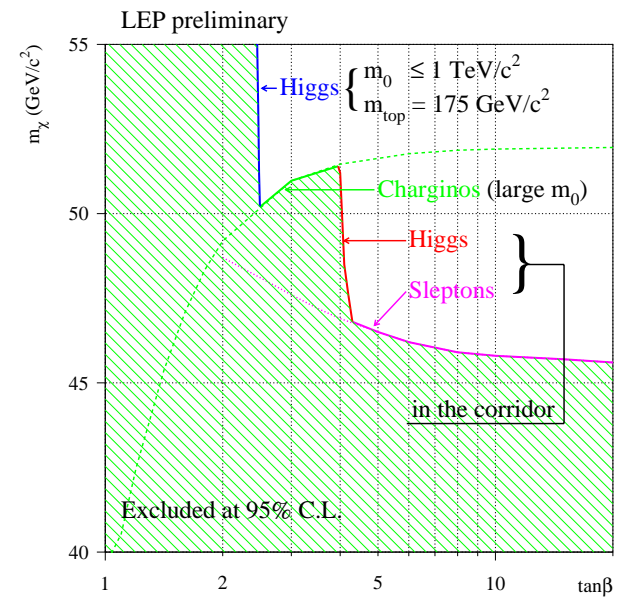

Figure 16: $95 \%$ C.L. lower limit on the mass of the lightest neutralino, as a function of $\tan \beta$, as obtained in the constrained MSSM by combining the four LEP experiments [1] $\left.{ }_{1}^{1}\right]$. The searches used to set the limit in the various $\tan \beta$ regions are indicated.

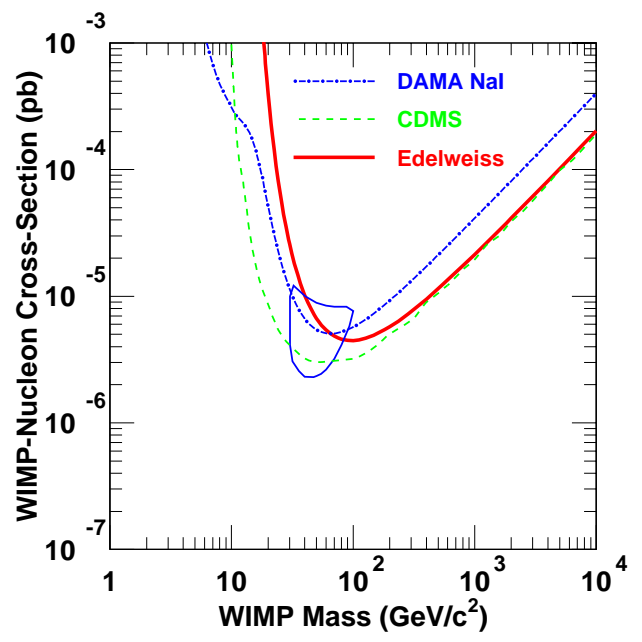

Figure 17: Region of the plane WIMP mass versus WIMP-nucleon cross-section favoured at the $3 \sigma$ level by the Dama experiment (closed contour, [23in). The regions excluded at $90 \%$ C.L. by the Edelweiss experiment (solide line, [24]), the CDMS experiment (dashed line, [25] sult (dash-dotted line, [2] $\left.{ }^{2} \bar{b}_{1}^{\prime}\right)$ are also shown.

The LEP bound on the LSP mass can be compared to the results of direct searches for cold dark matter. These are performed by underground experiments looking for neutralinos or, more generally, for Weakly-Interacting Massive Particles (WIMP's), coming from the galactic halo and interacting with the detectors via WIMP-nuclei scattering. One of these experiments, the DAMA experiment at Gran Sasso, has reported an annual modulation in the rate of nuclear recoils [2] $23 \overline{3}]$, which could be interpreted as due to galactic neutralinos. The region favoured by the DAMA observation, as well as the negative results from other experiments of similar scope, is shown in figure $1 \overline{1} \overline{7}_{-1}$ It can be seen that the LEP limit quoted above, which does not depend on the neutralino-nucleon cross-section, rules out about half of the region preferred by the DAMA data.

\subsubsection{Indirect constraints}

More model-dependent constraints on Supersymmetry can be obtained from results and arguments other than direct searches at Colliders. Examples derived in the framework of

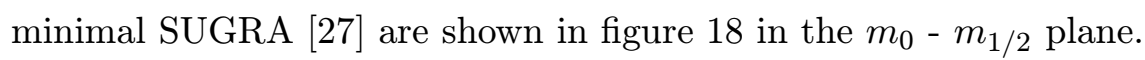

If SUSY particles are light, $b \rightarrow s \gamma$ decays can proceed through SUSY loops involving for instance squarks and charginos. The measurements [2] 
decay from CLEO and BELLE (a combined value $B R(b \rightarrow s \gamma)=(3.2 \pm 0.5) \cdot 10^{-4}$ has been used to derive the limit in figure $\left.{ }_{1}^{1} \underline{1}_{1}^{\prime}\right)$, in good agreement with the SM expectation, disfavour regions of the parameter space characterised by small sparticle masses.

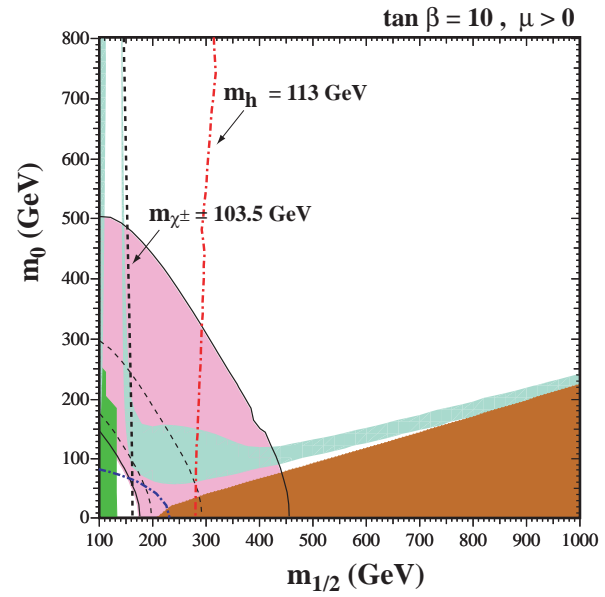

Figure 18: The minimal SUGRA plane $m_{0}$ $m_{1 / 2}$ for $\tan \beta=10, \mu>0$ and $A_{0}=0$ [2 $[2 \overline{7} \overline{1}]$. The vertical dashed line shows the limit from chargino searches at LEP, the vertical dot-dashed line an old limit from $h$-boson searches at LEP and the dot-dashed curve the limit from slepton searches at LEP. The dark-shaded (brown) region is theoretically forbidden, the medium-shaded (dark green) region is disfavoured by $b \rightarrow s \gamma$, and the light-shaded (light green) region is favoured by cosmology. The shaded (pink) region limited by solid black lines is favoured by the $g_{\mu}-2$ measurement at $2 \sigma$ (the dashed lines indicate the $\pm 1 \sigma$ contour).

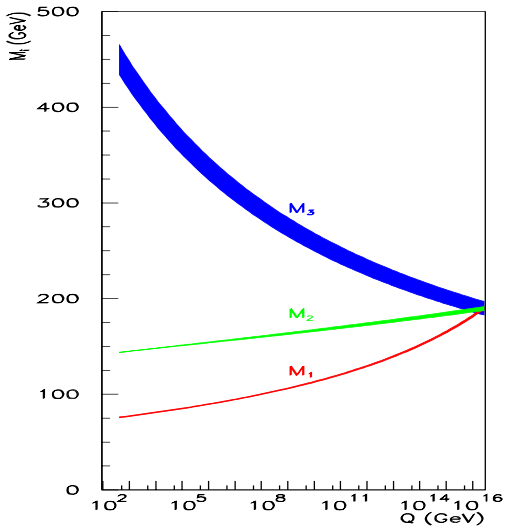

Figure 19: Evolution of the gaugino mass parameters $M_{1}, M_{2}$ (which are related to the neutralino and chargino masses) and $M_{3}$ (related to the gluino mass) from the measured values at the electroweak scale (with experimental accuracies reflected by the widths of the bands) up to the Grand Unification scale [32]. The chosen point in the minimal SUGRA parameter space is $m_{1 / 2}=190 \mathrm{GeV}$, $m_{0}=200 \mathrm{GeV}, \tan \beta=30, A_{0}=550 \mathrm{GeV}$, $\mu<0$.

Also shown in figure $1 \overline{1} \overline{1}_{1}^{\prime}$ is the region preferred by the recent measurement of the anomalous magnetic moment of the muon $g_{\mu}-2$ by the BNL E821 experiment [i2 $\left.2 \overline{1}\right]$, assuming that the reported $2.6 \sigma$ deviation from the SM prediction ${ }^{2}$ comes from SUSY contributions, e.g. loops involving charginos and sneutrinos.

Finally, the region preferred by cosmology (cold dark matter) has been derived assuming that the neutralino relic density be in the range $0.1 \leq \Omega_{\chi} h^{2} \leq 0.3$, where $h$ is the present Hubble expansion rate (in units $100 \mathrm{~km} / \mathrm{s} / \mathrm{Mpc}$ ) and $\Omega_{\chi}$ is the neutralino density normalised to the critical density of the universe.

\footnotetext{
${ }^{2}$ It should be noted that very recently, after the 2001 Summer Conferences, the theoretical calculations have been revisited [30]. As a consequence, the deviation of the BNL measurement from the SM expectation has decreased from $2.6 \sigma$ to $1.6 \sigma$.
} 
Figure $\overline{1} \overline{1} \overline{8}$ should not be considered as a collection of stringent limits, since it is based on many specific assumptions. However, it illustrates nicely the interplay between direct searches, precise measurements of propagator physics and cosmology. For instance, it is interesting to notice that there is a region, not excluded by present search results at Colliders, which is favoured by all the above-mentioned indirect measurements and predictions. In this region, squarks are lighter than $\sim 800 \mathrm{GeV}$, and are therefore accessible at future machines.

\subsubsection{The future}

The ultimate mass reach of the CDF and D0 experiments in Run 2 is about $200 \mathrm{GeV}$ for charginos and for stop squarks, about $250 \mathrm{GeV}$ for sbottom squarks and up to $450 \mathrm{GeV}$

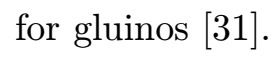

The LHC discovery potential extends up to squark and gluino masses of 2.5-3 TeV. Therefore, if nothing will be found at the LHC low-energy Supersymmetry will lose most of its motivation, for instance the possibility of stabilising the Higgs boson mass without much fine-tuning.

On the other hand, if SUSY will be found at the Tevatron or at the LHC, then a Linear Collider of sufficient centre-of-mass energy should be able to perform precise measurements of almost all kinematically accessible sparticles. In particular, chargino and neutralino masses should be measured to $0.1 \%$ [9. These results, combined with a measurement of the gluino mass at the LHC with an accuracy at the percent level, should provide insight of the structure of the theory at high energy, allowing for instance an accurate reconstruction of the common gaugino mass $m_{1 / 2}$. As shown in figure ${ }_{1}^{1} \bar{g}_{1}^{\prime}$, this should be possible by starting from the values of the gaugino masses measured at the electroweak scale and evolving them up to the Grand Unification scale by means of the Renormalisation Group

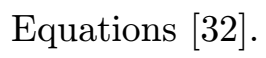

\section{Extra-dimensions}

These theories have raised a large interest over the last three years, with more than 700 theoretical papers published on the subject. Since most of the existing experimental results have been derived in the framework of ADD models (Arkani-Hamed, Dimopoulos, Dvali,

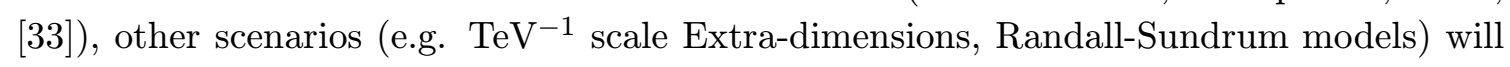
not be discussed here.

The basic idea of ADD theories (see [1] 10 in] for a more rigorous discussion) is that, if there exist $n$ additional spatial dimensions, and gravity is allowed to propagate in $4+n$ dimensions whereas the Standard Model world is confined in a 4-dimensional "wall", then the gravity scale (here called $M_{S}$ ) can be as low as $\sim 1 \mathrm{TeV}$, i.e. of the same order as the electroweak scale, thereby solving the hierarchy problem.

A first constraint to this scenario comes from the requirement that Newton's law in $4+n$ dimensions has to be equal to the standard 4-dimensional law at the (large) distances that are experimentally tested. This implies that the following relation must be satisfied 


$$
M_{P l}^{2} \approx M_{S}^{n+2} R^{n}
$$

between the Planck scale $M_{P l}$, the new gravity scale $M_{S}$, the number $n$ of extradimensions and their physical size $R$. Assuming $M_{S} \approx 1 \mathrm{TeV}$, then only one extradimension is excluded, because its size should be $R \approx 10^{13} \mathrm{~cm}$ from the above formula, which is ruled out by macroscopic observations. But when $n$ increases, the size $R$ of the extra-dimensions must decrease according to equation $\underline{\overline{5}} . \overline{1}_{j}$ and already for $n=2$ becomes smaller than the sensitivity of present gravity experiments, which are able to test Newton's law down to $\approx 1 \mathrm{~mm}$. This shows that the gravity scale could be as low as $\sim 1 \mathrm{TeV}$, without contradicting the existing experimental constraints, provided that there exist $n$ extra-dimensions compactified at the sub-mm level.

An important consequence of these theories is that gravitons propagating in compact extra-dimensions of size $R$ acquire, by virtue of the Heisenberg principle, a quantised mass $m_{k} \sim k / R$, where $k$ is an integer number. And since the spacing between adjacent energy levels is predicted to be small ( $\delta m \sim 1 / R$, e.g. $400 \mathrm{eV}$ for $n=3)$, these gravitons give rise to a continuous tower of massive states called "Kaluza-Klein excitations".

The graviton production cross-section, for instance for two incoming fermions, can therefore be written as

$$
\sigma(f f \rightarrow G) \approx \frac{1}{M_{P l}^{2}} N_{k k}
$$

where $1 / M_{P l}$ is the coupling at the $f f G$ vertex, and $N_{k k}$ is the number of gravitons which can be produced in the interaction. The latter depends on the available phase space and is therefore proportional to the centre-of-mass energy divided by the spacing between Kaluza-Klein levels: $N_{k k} \sim(\sqrt{s} / \delta m)^{n}$. Using the relation $\delta m \sim 1 / R$ and equation 5.1 , one obtains:

$$
\sigma(f f \rightarrow G) \approx \frac{1}{M_{P l}^{2}} \sqrt{s}^{n} R^{n} \approx \frac{\sqrt{s}^{n}}{M_{S}^{n+2}}
$$

That is, due to the large number of available Kaluza-Klein gravitons, the effective coupling between SM particles and gravitons becomes of detectable electroweak strength $\left(M_{S} \sim 1 \mathrm{TeV}\right.$ in the above formula). This scenario is very attractive because it implies there is only one scale in particle physics, the electroweak scale, and that it should be possible to test quantum gravity and the geometry of the universe in our laboratories.

Present constraints on Extra-dimension theories come from astrophysics and cosmology, table-top gravity experiments and Colliders.

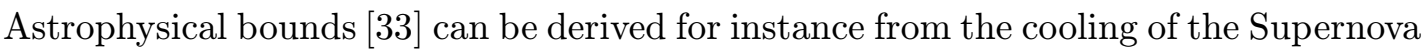
SN1987A, which is due to neutrino emission, as measured by the IMB and Superkamiokande experiments in agreement with the predictions. This sets constraints on additional cooling via graviton emission, which translates into the limits $M_{S}>31 \mathrm{TeV}(2.7 \mathrm{TeV})$ for $n=2$ $(n=3)$. Although there are in general large uncertainties on these astrophysical bounds, $n=2$ is clearly disfavoured if $M_{S}$ is required to be of the order of $1 \mathrm{TeV}$. 
The most stringent constraints from gravity tests at short distances come from the Seattle experiment [3. and a rotating attractor down to distances of $\sim 200 \mu \mathrm{m}$. No deviations have been observed from Newton's law, and the limit $M_{S}>1.9 \mathrm{TeV}$ for $n=2$ has been set.

Smaller distances can only be explored by high-energy Colliders. It should be noted, however, that no constraints are provided by precision measurements for instance of electroweak observables at LEP, because the contribution of graviton loops to these observables are suppressed by a factor $\left(m_{Z} / M_{S}\right)^{n+2}$. For $n \geq 2$ this factor is of order $10^{-4}$, i.e. much smaller than e.g. the typical contributions expected from SUSY loops.

On the other hand, direct searches for graviton production at high-energy Colliders are powerful because, as shown in equation 5.2, , the cross-section increases with increasing centre-of-mass energy. Only results from LEP are available for the time being. At this machine, gravitons can be produced in association with a photon through the process $e^{+} e^{-} \rightarrow \gamma^{*} \rightarrow \gamma G$. The expected signature is a single photon plus missing energy, since gravitons escape detection. For this type of final state, figure $2 \overline{0}_{1}^{\prime}$ shows the distribution of the event missing mass as obtained by DELPHI. A signal from graviton production would show up at large values of the missing mass since gravitons are expected to be heavy. Since no excess has been found, neither by DELPHI nor by the other LEP experiments, lower limits on $M_{S}$ have been set which range from $\sim 1 \mathrm{TeV}$ for $n=2$ to $\sim 0.6 \mathrm{TeV}$ for $n=6$. Similar bounds have been set at LEP, Tevatron and HERA by indirect searches, i.e. by looking for deviations from the SM expectation of the measured cross-sections and particle angular distributions in various final states. Deviations could be a sign of exchange of virtual gravitons between the incoming and the outgoing particles.

The approximate potential of future Colliders for the investigation of ADD Extradimension theories is summarised in figure $\overline{2} \overline{1}$. The present lower limit on the gravity scale is about $1 \mathrm{TeV}$ for $n=3$. For the same number of extra-dimensions, the Tevatron Run 2 has a reach of about $2 \mathrm{TeV}$ on $M_{S}$ with only $2 \mathrm{fb}^{-1}$ of integrated luminosity, and TESLA can achieve 5-6 TeV with $500 \mathrm{fb}^{-1}$. The LHC has a sensitivity of up to $9 \mathrm{TeV}$ for $100 \mathrm{fb}^{-1}$, thanks to the strong enhancement of graviton effects at high energy. If nothing will be found at the LHC, also these theories, like Supersymmetry, will lose most of their motivation and appeal, although it is possible to evade the experimental limits by increasing the number of extra-dimensions.

\section{Flavour-changing neutral current processes}

One of the challenges of any theory beyond the Standard Model is to account for the very low observed rate of FCNC processes, that in the Standard Model are forbidden at the tree level. At the same time these processes are an obvious place to look for New Physics, since there the SM background is small. The top sector is particularly interesting because the amplitude of FCNC processes is related to the mass differences between quarks, and therefore deviations from the Standard Model could be first observed in channels involving the top quark. 


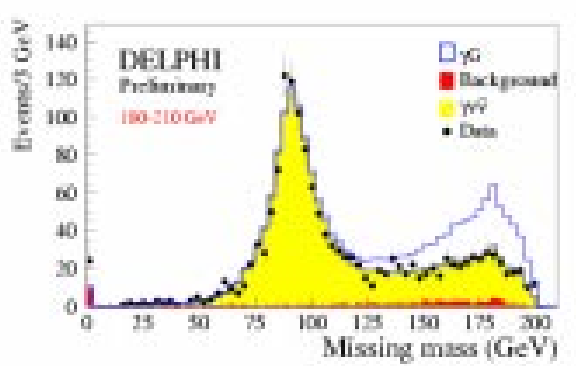

Figure 20: The distribution of the missing mass in events with photons plus missing energy from DELPHI [35i]. The dots with error bars are the data, the light-shaded region shows the expectation from the dominant SM background ( $\gamma \nu \bar{\nu}$ production), the dark-shaded region the contribution from other backgrounds, and the solid line the expected signal from graviton production for $M_{S}=0.75 \mathrm{TeV}$ and $n=2$.

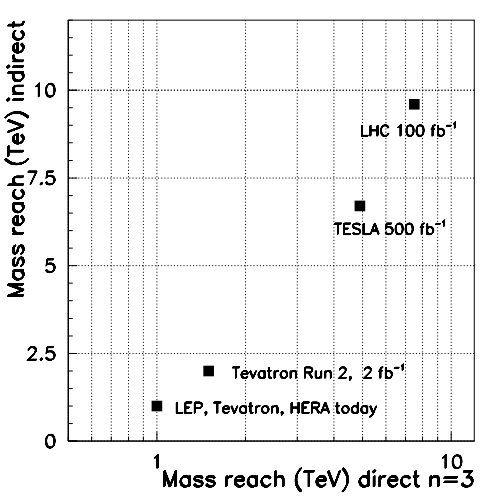

Figure 21: In the framework of ADD models, the $95 \%$ C.L. reach on the gravity scale $M_{S}$ from indirect searches and from direct searches (for $n=3$ ) at present and future Colliders.

For these reasons all three machines, LEP, Tevatron and HERA, have looked for the FCNC vertices where a top quark transforms into a charm or a up quark by emitting a photon or a $Z$ (an example is shown in figure $2 \overline{2} 2$ ). The amplitudes at the $t q \gamma$ and $t q Z$ vertices are governed by the parameters $k_{\gamma}$ and $k_{Z}$ respectively. CDF has looked for the FCNC top decays $t \rightarrow Z q, \gamma q$ (branching ratio smaller than $10^{-10}$ in the SM) in $t \bar{t}$ final states. The LEP experiments have looked for single-top production in association with a charm or a up quark (figure $\left.\overline{2}_{2} \overline{2}_{1}\right)$. The HERA experiments have also looked for single-top production but, unlike the LEP experiments, they can only probe the $t u \gamma$ vertex, through the neutral-current $\gamma$-exchange process $e^{ \pm} u \rightarrow e^{ \pm} t$, and therefore can only constrain $k_{\gamma}$. The negative results of these searches have been translated into exclusion regions in the $k_{\gamma}-k_{Z}$ plane (figure $\left.2 \overline{2}^{-1}\right)$. The presently unexcluded region (approximately $k_{Z}<0.2$ and $\left.k_{\gamma}<0.3\right)$ corresponds to top branching ratios into $u$-quark and $c$-quark of a few percent.

\section{The " $W$ " excess in $\mathbf{H 1}$}

The HERA limit on single-top production shown in figure $2 \overline{2} \overline{3}_{1}^{1}$ is that obtained by ZEUS. $\mathrm{H} 1$ has also performed a similar search, but the resulting bound is less stringent. This is

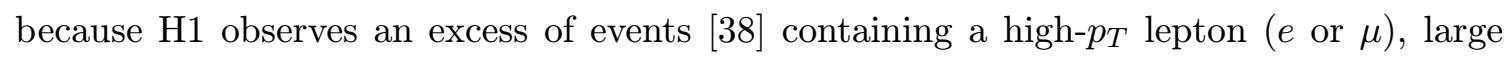
missing transverse energy and a high- $p_{T}$ hadronic system, a topology expected for instance from $W$ production [ī $[1 \overline{2}]$, as well as from single-top production. 


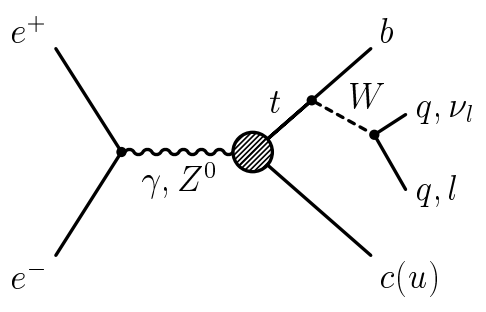

Figure 22: Feynman diagram for single-top production at LEP. The FCNC $t q \gamma / t q Z$ vertex is shown as a blob (courtesy of A. Leins).

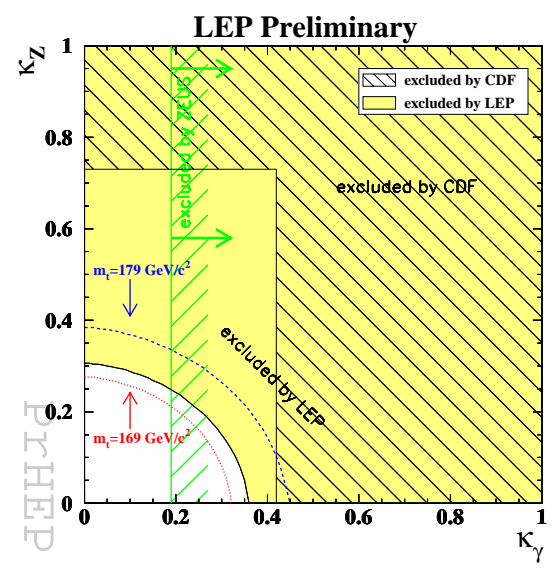

Figure 23: Regions of the $k_{\gamma}-k_{Z}$ plane excluded at $95 \%$ C.L. by searches for FCNC top couplings. The hatched region is ex-

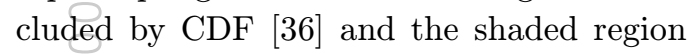
by LEP (the effect of varying the top mass by $\pm 1 \sigma$ of the measured value is also indicated) [3근. The vertical line shows the bound from ZEUS [38 $\left.\bar{B}^{1}\right]$.
Figure $2 \overline{2} \overline{4}$ shows that, for high values of $p_{T}^{X}$, the $p_{T}$ of the hadronic system, more events are observed in the data than they are predicted by the Standard Model. For instance, for $p_{T}^{X}>25 \mathrm{GeV}(40 \mathrm{GeV})$, ten (six) events are observed in $102 \mathrm{pb}^{-1}$ of data, with $2.82 \pm 0.73(0.99 \pm 0.28)$ expected from SM processes. The dominant SM contribution (more than $80 \%$ of the total) comes from $W$ production. On the other hand, no excess has been found by ZEUS in these final states in a data sample of $130 \mathrm{pb}^{-1}$. It should be noted that both experiments agree on their estimates for the Standard Model expectation.

The origin of the spectacular $\mathrm{H} 1$ events in the high- $p_{T}^{X}$ tails of the distribution in figure $2 \overline{2}^{4}$, besides a statistical fluctuation, is presently under investigation. Single-top production, which gives rise to a similar signature if the top decays semileptonically $t \rightarrow b \ell \nu$, is unlikely, because the excess in this channel is not significant (five events observed with $1.8 \pm 0.5$ expected) and no excess is observed in the fully-hadronic $t \rightarrow b j j$ final states. Anomalous Triple Gauge Couplings (some of the $W$ production diagrams involve $W W \gamma$ and $W W Z$
H1 PRELIMINARY 101.6ph ${ }^{-1} \mathrm{e}^{+} \mathrm{p}$ data 94-00
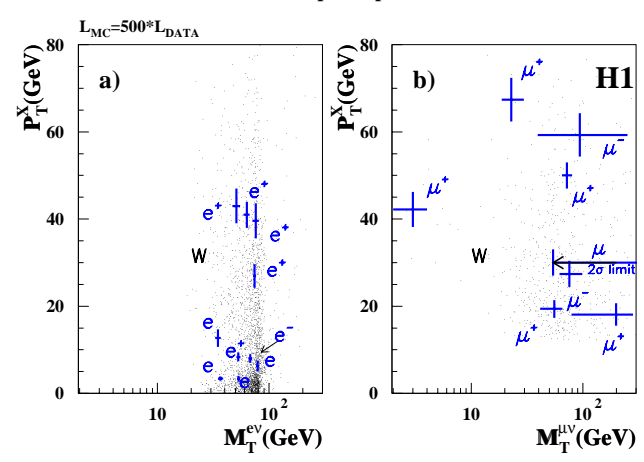

Figure 24: The distribution of the $p_{T}$ of the hadronic system $p_{T}^{X}$, as a function of the transverse mass of the leptonmissing energy system, for the $\mathrm{H} 1$ " $W$ sample". The crosses show the data, whereas the dots show the SM expectation for an integrated luminosity 500 times larger than that of the data [3isin'. 
vertices) are excluded by LEP, which has a much larger sensitivity to these couplings. Other interpretations in terms of New Physics, e.g. R-parity violating production of SUSY particles or excited quarks, are being evaluated. The new data which will be collected with the upgraded machine starting in fall 2001 should shed light on this question.

\section{Conclusions}

The exceptional LEP era, which has recently come to a conclusion, has produced three main results in the domain of searches. First, the Higgs boson is heavier than $114.1 \mathrm{GeV}$. However, a $2 \sigma$ hint and a few beautiful candidates at a mass of about $115 \mathrm{GeV}$, as well as the fit to the electroweak data $\left(m_{H}<196 \mathrm{GeV}\right.$ at $95 \%$ C.L., [1] $\left.\left.{ }_{2}^{1}\right]\right)$, suggest that the Higgs could be just around the corner. It should be noted that, although theories predicting a heavy Higgs boson (e.g. $m_{H} \sim 500 \mathrm{GeV}$ ) can technically still be consistent with the

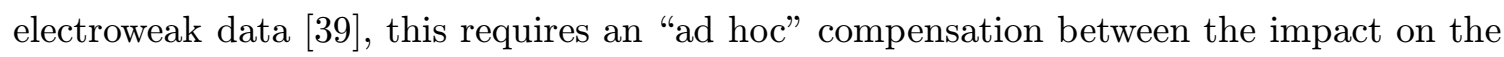
data from the Higgs sector and from other new particles (e.g. a new $Z^{\prime}$ ). Second, in general direct searches have excluded new particles with masses up to $\sim 100 \mathrm{GeV}$, if pair-produced like SUSY particles, and up to $\sim 200 \mathrm{GeV}$ if singly-produced. Third, by combining and interpreting the results of several searches (e.g. Higgs and SUSY), it has been possible to put very stringent constraints in the parameter space of minimal models, and to derive a lower limit of about $45 \mathrm{GeV}$ on the LSP mass within the CMSSM. For these reasons, the outcomes of ten years of experimentation at LEP represent a real challenge for New Physics.

The Tevatron Run 2 will pursue, with increased sensitivity, the exploration of the few hundred GeV region started in Run 1, with a discovery reach of up to $\sim 1 \mathrm{TeV}$ in some cases (e.g. a $Z^{\prime}$ boson). If an integrated luminosity of $\sim 15 \mathrm{fb}^{-1}$ will be collected, CDF and D0 will have good chances to discover a SM Higgs boson up to masses of $\sim 120 \mathrm{GeV}$ or to exclude it (at $95 \%$ C.L.) up to masses of $\sim 185 \mathrm{GeV}$, thereby covering most of the range preferred by the electroweak data.

The upgraded HERA has a mass reach of up to $\sim 300 \mathrm{GeV}$ from direct searches, and is complementary to Tevatron in some respects, e.g. in the sensitivity to leptoquarks and to R-parity violating SUSY. There is an intriguing excess in the H1 data which needs to be understood.

At the end of the decade, the LHC will explore in depth the highly-motivated energy range of up to a few $\mathrm{TeV}$, and therefore will say the final word about the existence of a SM Higgs boson (if not yet discovered at that time), Supersymmetry, and other TeV-scale predictions.

\section{Acknowledgments}

The results presented in this paper are based on the work of many people, and it would be impossible to thank them all. I am particularly grateful to the LEP Higgs Working Group and the LEP SUSY Working Group, and to their convenors P. Igo-Kemenes and L. Pape. 
I would also like to thank F. Cerutti, B. Clerbaux, M. Felcini, P. Janot, T. Junk, M. Kado, M. Kuze, E. Perez, M. Spiropulu and S. Wynhoff.

\section{References}

[1] P. Igo-Kemenes, LEPC Open Session, 3 November 2000, http://lephiggs.web.cern.ch/LEPHIGGS/talks/index.html.

[2] LEP Higgs working group, CERN-EP/2001-005, [hep-ex/0107029].

[3] ALEPH Collaboration, 'Ph hys. Lett. B

[4] DELPHI Collaboration, iPhys. Lett. B $4999(2001) 23$.

[5] L3 Collaboration, "Phys. Lett. B $\mathbf{5} 1 \overline{1} \overline{2}(2001) 319^{\prime}$

[6] OPAL Collaboration, iPhys. Lett. B 49992001$) 38$.

[7] M. Carena, J.S. Conway, H.E. Haber, J.D. Hobbs, et al., Report of the Higgs Working Group for Run 2 of the Tevatron, [hep-ph/0010338].

[8] ATLAS Collaboration, Detector and physics performance Technical Design Report, CERN/LHCC/99-15.

[9] R. Heuer et al., TESLA Technical Design Report, [hep-ph/0106351].

[10] P. Binetruy, these Proceedings.

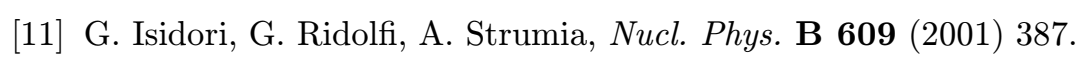

[12] D. Charlton, these Proceedings.

[13] M. Masip, íphys. L

[14] LEP Higgs working group, LHWG Note/2001-04, [hep-ex/0107030].

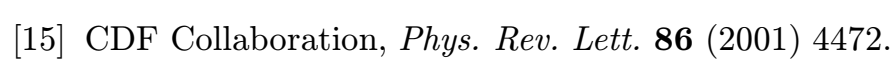

[16] LEP SUSY working group, http://lepsusy.web.cern.ch/lepsusy/.

[17] CDF Exotics Physics group, http://www-cdf.fnal.gov/physics/exotic/exotic.html.

[18] D0 Physics group on Searches for New Phenomena, http://www-d0.fnal.gov.

[19] CDF Collaboration, FERMILAB-PUB-01/084-E, submitted to Phys. Rev. Lett..

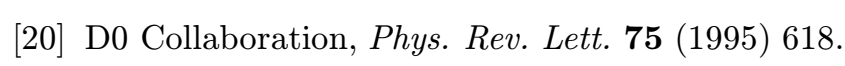

[21] L3 Collaboration, L3 note 2644, March 2001.

[22] ALEPH Collaboration, iPhys. Lett.

P. Azzurri, these Proceedings.

[23] R. Bernabei et al., 'Physs. Lett.

[24] A. Benoit et al., [astro-ph/0106094].

[25] R. Abusaidi et al., Physs. Rev. Lett. 84 (2000) 5699 .

[26] R. Bernabei et al., iPhys. Lett. B $\mathbf{3} 8 \mathbf{9}(1996) 75 \overline{7}$.

[27] M. Battaglia et al., CERN-TH/2001-150, [hep-ph/0106204]. 
[28] S. Ahmed et al., CLEO CONF 99-10;

BELLE Collaboration, BELLE-CONF-0003.

[29] H. N. Brown et al., ,'Phys. Rev. Lett. 86 $(2001) 2227$ t.

[30] M. Hayakawa and T. Kinoshita, KEK-TH-793, [hep-ph/0112102].

[31] V. Barger, C.E.M. Wagner, et al., Report of the SUGRA Working Group for Run 2 of the Tevatron, [hep-ph/0003154].

[32] G.A. Blair, W. Porod and P.M. Zerwas, 1 Phys. Rev. $\mathbf{D} \mathbf{6} \overline{3}(2001) 017 \overline{0} \overline{3}$.

[33] N. Arkani-Hamed, S. Dimopoulos and C. Dvali, Lhys. Lett. B. $\mathbf{4} \mathbf{2} 9(1998) 263$.

[34] E. Adelberger et al., ,'Phys. Rev. Lett. 86 (2001) 1418:

[35] DELPHI Collaboration, A. Anashkin et al., DELPHI 2001-011 CONF 452.

[36] CDF Collaboration, Phys. Rev.Lett. 80_(1998)_2525.

[37] M. Pimenta, these Proceedings.

[38] C. Vallée, these Proceedings.

[39] M. Peskin and J. Wells, ${ }_{\text {Phys. Rev. D } 64}$ (2001) 093003 : 